\title{
Pulmonary hypertension and hepatic encephalopathy: lethal complications of Rendu-Osler-Weber disease
}

\author{
${ }^{1} \mathrm{TJ}$ Ford, ${ }^{2} \mathrm{MW}$ Fong, ${ }^{3} \mathrm{BC}$ Cheah, ${ }^{4} \mathrm{C}$ Alexopolous \\ ${ }^{1}$ Advanced Trainee in Cardiology; ${ }^{2}$ Basic Physician Trainee, Prince of Wales Hospital, Sydney, Australia; ${ }^{3}$ Medical Student, University of New \\ South Wales, Sydney; ${ }^{4}$ Consultant Cardiologist, Port Macquarie Base Hospital, Port Macquarie, Australia
}

\begin{abstract}
Hereditary haemorrhagic telangiectasia (HHT) is an autosomal dominant disorder characterised by epistaxis, mucocutaneous telangiectasia with systemic manifestations due to visceral telangiectasia and arterio-venous malformations (AVMs). We describe unusual complications of HHT in a 68-year-old male who developed high-output cardiac failure with pulmonary hypertension in combination with hepatic encephalopathy due to hepatic AVMs. This case shows the importance of a multi-system approach to HHT and considers the treatment of its hepatic complications.
\end{abstract}

Correspondence to TJ Ford Prince of Wales Hospital Sydney, NSW 2031

Australia

tel. +6I 426267347

e-mail t.ford@unsw.edu.au

KEYWORDS hereditary haemorrhagic telangiectasia, hepatic arteriovenous malformation, hepatic encephalopathy, high-output cardiac failure, pulmonary hypertension

DECLARATION OF INTERESTS No conflict of interest declared.

Hereditary haemorrhagic telangiectasia $(\mathrm{HHT})$ is an autosomal dominant disorder characterised by epistaxis, mucocutaneous telangiectasia with systemic manifestations due to arterio-venous malformations (AVMs) and visceral telangiectasia. First described in 1864 by Henry Gawen Sutton,' the triple eponym 'Rendu-Osler-Weber disease' was appended at the turn of the twentieth century following sequential published case series. ${ }^{2}$

The hallmark telangiectasias are focused expansions of post-capillary venules together with AVMs. Clinical features depend on the affected sites and include epistaxis, bleeding diathesis, iron-deficiency anaemia, stroke, respiratory and cardiac complications. We report a case with rare sequelae of hepatic AVMs: encephalopathy from porto-systemic shunting together with high-output cardiac failure (HOCF), resulting in pulmonary hypertension $(\mathrm{PH})$. This case highlights the need for multisystem assessment to optimise management of this disorder. Awareness and recognition of these complications are relevant given therapeutic developments in the management of both $\mathrm{PH}$ and liver disease in HHT.

\section{CASE REPORT}

A 68-year-old male presented with a one-week history of progressive drowsiness, dyspnoea (New York Heart Association Class III) and lower limb oedema. The patient was diagnosed with HHT in his teens following recurrent severe epistaxis. His disease was complicated by chronic gastrointestinal blood loss and iron-deficiency anaemia. Three years previously he was found to have vigorous left ventricular systolic function with a

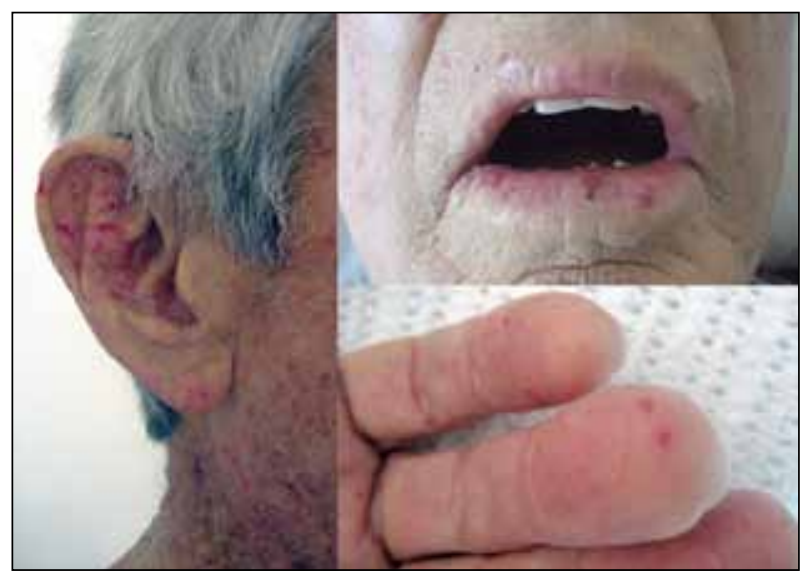

FIGURE I Widespread telangiectasia distributed over the ear and face (left), lips (top right) and finger pulps (bottom right).

suggestion of moderately elevated estimated pulmonary artery pressure on echocardiography.

Investigations into his pulmonary hypertension and high output cardiac state at that time revealed no evidence of pulmonary thromboembolic disease on lung perfusion imaging but a large hepatic AVM on abdominal ultrasound. He was reluctant to undergo invasive investigations or treatment of the latter. In addition, he had recently been commenced on therapy for the chronic phase of chronic myeloid leukaemia detected on routine blood examination one month earlier. The patient was independent, a life-long non-smoker with no history of alcohol abuse. His regular medications included tranexamic acid, imatinib, metoprolol, frusemide, spironolactone and allopurinol. 
TABLE I Laboratory results (Reference range in brackets)

\begin{tabular}{|l|l|}
\hline $\mathrm{Hb}$ & $92 \mathrm{~g} / \mathrm{L}(\mathrm{I} 20-160 \mathrm{~g} / \mathrm{L})$ \\
\hline $\mathrm{MCV}$ & $\mathrm{I} 0 \mathrm{IfL}(80-100 \mathrm{fL})$ \\
\hline Hematocrit & $29 \%(40-54)$ \\
\hline Platelet & $294 \times 109 / \mathrm{L}(\mathrm{I} 50-400 \times 109 / \mathrm{L})$ \\
\hline White cell count & $5.9 \times 109 / \mathrm{L}(4-\mathrm{II} \times 109 / \mathrm{L})$ \\
\hline INR & 1.4 \\
\hline Na I33 & $\mathrm{K}+5.4$ \\
\hline Urea & $27.5 \mathrm{mmol} / \mathrm{L}(2.9-7 . \mathrm{I})$ \\
\hline Creatinine & $250 \mu \mathrm{mol} / \mathrm{L}(6 \mathrm{I} .9-\mathrm{II} 5)$ \\
\hline AST & $33 \mathrm{U} / \mathrm{L}(<35)$ \\
\hline ALT & $16 \mathrm{U} / \mathrm{L}(<35)$ \\
\hline ALP & $549 \mathrm{U} / \mathrm{L}(36-92)$ \\
\hline GGT & $515 \mathrm{U} / \mathrm{L}(<30)$ \\
\hline Bilirubin & $54 \mu \mathrm{mol} / \mathrm{L}(<20)$ \\
\hline Albumin & $31 \mathrm{~g} / \mathrm{L}(35-55 \mathrm{~g} / \mathrm{L})$ \\
\hline Ammonia & $190 \mu \mathrm{mol} / \mathrm{L}(<50)$ \\
\hline
\end{tabular}

Abbreviations:ALP: alkaline phosphatase;ALT: alanine aminotransferase; AST: aspartate aminotransferase; GGT: gammaglutamyl transpeptidase; $\mathrm{Hb}$ : haemoglobin; INR: international normalised ratio; $\mathrm{MCV}$ mean corpuscular volume; $\mathrm{U} / \mathrm{L}$ = units/litre

On examination, he was frail and drowsy (Glasgow Coma Scale 13). He was mildly icteric with prominent asterixis. There were no peripheral stigmata of chronic liver disease or signs of portal hypertension, but mucocutaneous telangiectasia were diffusely distributed (Figure I).

The jugular venous pressure was raised to the angle of the jaw with prominent $\mathrm{V}$-waves. The patient had gross dependent oedema extending to his thighs. The peripheral pulse was high volume and irregularly irregular with a rate of 100 beats per minute maintaining a blood pressure of 110/64. There was a prominent left parasternal heave, a loud pulmonary component to the second heart sound and a grade III pan-systolic murmur over the left sternal edge.

Respiratory examination revealed signs of a right pleural effusion together with bilateral basal crepitations. Abdominal examination was remarkable for signs of increased blood flow through the hepatic AVM with prominent pulsation and obvious bruit. There was no ascites and neurological examination did not reveal any localising neurological deficits.

Laboratory work-up (Table I) confirmed an abnormal but stable full blood count with renal impairment. There was a cholestatic pattern of liver impairment with mild derangement of markers of true hepatic synthesis. Serum ammonia was elevated while a liver screen was negative for hereditary haemochromatosis, viral hepatitis and autoimmune disease including normal thyroid function.

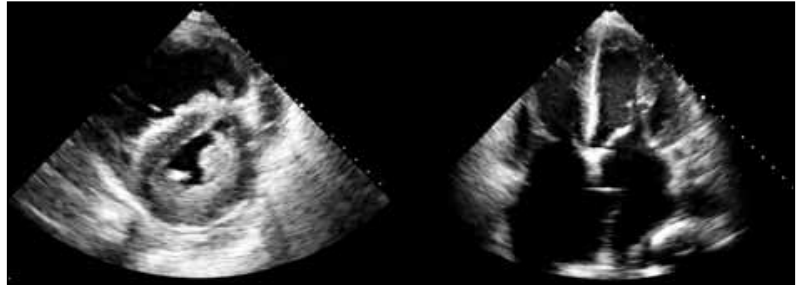

FIGURE 2 Transthoracic echocardiogram demonstrating interventricular septal flattening in systole, consistent with right ventricular pressure overload (short axis view; left) and an aneurysmal atrial septum with right to left bowing in keeping with raised right atrial pressure, with the right heart situated on the left and vice versa (four-chamber apical view; right).

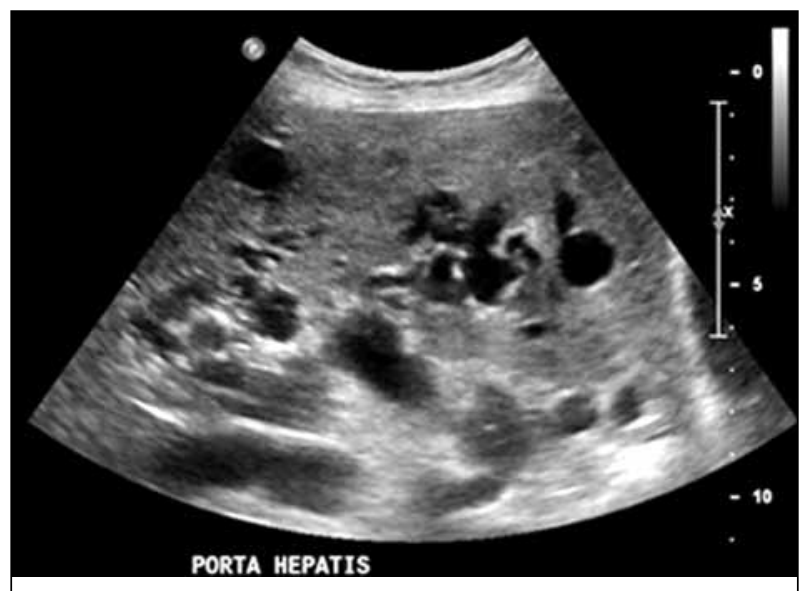

FIGURE 3 Liver ultrasound at the level of the porta hepatis demonstrating multiple tortuous vessels in keeping with a hepatic arteriovenous malformation.

An electrocardiogram revealed atrial fibrillation with a ventricular response rate of 90 beats per minute, together with long-standing right bundle branch block. A chest radiograph showed an enlarged cardiac silhouette, upper lobe venous diversion, increased interstitial lung markings and a right-sided pleural effusion consistent with pulmonary congestion. The patient's transthoracic echocardiogram (Figure 2) was in keeping with high-output cardiac failure, demonstrating hyperdynamic left ventricular systolic function, severe biatrial dilation, raised estimated cardiac output at $8.3 \mathrm{~L} /$ minute $\left(4.9 \mathrm{~L} / \mathrm{m}^{2}\right)$ [reference range $2.6-4.7 \mathrm{~L} / \mathrm{m}^{2}$ ] and severe tricuspid regurgitation with severe pulmonary hypertension (estimated pulmonary artery systolic pressure, PASP, of $73 \mathrm{mmHg}$ [reference range $<35$ $\mathrm{mmHg}]$ ). The PASP was likely underestimated in the setting of a dilated right ventricle with impaired systolic function.

The computed tomography brain scan was normal. Abdominal ultrasound demonstrated dilated hepatic arteries with tortuous vessels characteristic of his known hepatic AVM (Figure 3). There was normal liver echogenicity with a normal splenic size, patent portal vein, no biliary duct dilation and no evidence of hydronephrosis. 
An exhaustive search for the clinical suspicion of occult infection or gastrointestinal haemorrhage did not yield a cause for the acute deterioration. He was managed with fluid restriction, diuretics and empirical antibiotics while high-dose osmotic laxatives were used in an attempt to improve his encephalopathy. Despite aggressive medical management, his clinical status deteriorated with worsening encephalopathy and cardio-respiratory failure. After discussion with the family and in accordance with the patient's advanced directive, a palliative approach was implemented and he died ten days after admission.

\section{DISCUSSION}

The development of hepatic AVMs is a common but under-recognised feature of $\mathrm{HHT}^{3}$ Depending on the mode of imaging assessment, the prevalence of hepatic AVMs in HHT clinic populations is around $40 \%$ using hepatic ultrasound or as high as $74 \%$ using computed tomography. ${ }^{4.5}$ While more than three-quarters of patients with hepatic AVMs are asymptomatic, screening for them is advocated by some, given that the diagnosis and prognosis depends on the presence and extent of

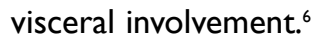

Patients with symptomatic hepatic AVMs typically present in one of three ways: HOCF, portal hypertension and biliary disease. ${ }^{7}$ We identified a number of contributory factors to our patient's deranged liver function: portal hypertension with hepatic venous congestion resulting from heart failure, arteriovenous shunting, portovenous shunting with resultant cholangiopathy.

The diagnosis of hepatic encephalopathy was made on the basis of an encephalopathic syndrome without focal neurology and was supported by the normal CT brain and raised plasma ammonia. Laboratory results (including only mildly impaired indices of true hepatic function) indicated a degree of cirrhosis; however, the relatively normal ultrasound and other laboratory results would make advanced cirrhosis unlikely. Hence, we hypothesise that hepatic encephalopathy resulted from a combination of intrinsic liver disease together with portosystemic shunting through the hepatic AVM. This significant complication may occur in HHT despite only mildly abnormal liver function tests. This is an important consideration when patients with HHT present with delirium, sleep-wake disturbances or other unexplained neurological symptoms. ${ }^{8}$

While encephalopathy as a result of hepatic AVMs is a rare complication of $\mathrm{HHT}$, its prompt recognition allows appropriate treatment with osmotic laxatives and consideration of procedures for shunt reduction. Shunt reduction may be achieved either by trans-arterial embolisation or hepatic artery surgical ligation, depending on patient anatomy and clinical condition.?
The benefits of hepatic artery occlusion are transient and associated risks include ischaemic cholangitis, ischaemic cholecystitis with or without hepatic necrosis. Such events necessitate liver transplantation or result in death in $30 \%$ of treated cases. ${ }^{9}$ Liver transplantation may be curative in patients unresponsive to medical treatment. However, our patient had advanced disease, poor functional status and no wish for invasive tests or treatments and hence this was not considered as an option.

There are systemic treatment approaches that have been used in HHT.Vascular endothelial growth factor is a key regulator of angiogenesis and is increased in patients with HHT. Bevacizumab, a monoclonal antibody to vascular endothelial growth factor, has been used with variable success as an anti-angiogenic drug in bleeding and hepatic complications of HHT. ${ }^{10,11}$ There is some evidence suggesting it may improve HOCF and epistaxis. ${ }^{12,13}$

Systemic therapy with the antifibrinolytic agent tranexemic acid has also been used with some efficacy in HHT and may have synergistic action with bevacizumab. ${ }^{14}$ Bevacizumab was not considered in our patient given his rapid preterminal decline, although it may have been used at an earlier stage in his illness.

Pulmonary hypertension in our patient was the result of HOCF secondary to the hepatic AVM. It is under-recognised in $\mathrm{HHT}$, and may be due to either left heart disease (as in this case) or less commonly due to increased pulmonary vascular resistance (pulmonary arterial hypertension, PAH). ${ }^{15-17}$ Pulmonary hypertension is more common in patients with symptomatic hepatic AVMs and may be explained by a higher prevalence of a high cardiac output state in this subgroup. Interestingly, while the recently commenced imatinib can decrease pulmonary pressure in patients with $\mathrm{PH}$, it may have contributed to his deterioration in HOCF and susceptibility to occult respiratory infection. ${ }^{18}$

Transthoracic echocardiography in our patient was consistent with HOCF, demonstrating hyperdynamic systolic function with a significantly raised estimated cardiac index. Confirmatory right heart catheterisation was not appropriate, given our patient's advanced directive and his co-morbidities. Distinguishing between aetiological types of $\mathrm{PH}$ is important, however, as this determines clinical management. High-output cardiac failure can be treated by reducing the cardiac output by reducing the blood flow through hepatic AVM shunt reduction procedures and conventional medical therapy, including optimising haemoglobin and treatment of cardiac arrhythmias. ${ }^{13}$ Identifying HHT patients with PAH may enable the use of novel advanced systemic therapies aimed at reducing pulmonary vascular resistance (e.g. prostanoids, endothelin receptor antagonists or phosphodiesterase 5 inhibitors). ${ }^{19}$ 


\section{KEY POINTS}

- Patients with HHT may develop visceral AVMs with severe systemic sequelae.

- In patients with $\mathrm{HHT}$, hepatic AVMs are common but under-recognised and have three main clinical consequences:

- High output cardiac failure +/- pulmonary hypertension

- Portal hypertension

- Biliary disease

- Awareness of the fatal complications that can occur in HHT with hepatic AVMs may enable earlier recognition and application of various novel treatments.

\section{REFERENCES}

I Hanes FM. Multiple hereditary telangiectases causing hemorrhage (hereditary hemorrhagic telangiectasia). Bull Johns Hopkins Hosp 1909; 20:63-73.

2 Fuchizaki U, Miyamori H, Kitagawa S et al. Hereditary haemorrhagic telangiectasia (Rendu-Osler-Weber disease). Lancet 2003; 362:1490-4.

3 Draghi F, Presazzi A, Danesino G et al. Hepatic sonography in patients with hereditary hemorrhagic telangiectasia hospitalized for epistaxis. J Ultrasound 2012; 15:164-70. http://dx.doi. org/I0.10I6/j.jus.2012.04.00I

4 Buscarini E, Leandro G, Conte D et al. Natural history and outcome of hepatic vascular malformations in a large cohort of patients with hereditary hemorrhagic teleangiectasia. Dig Dis Sci 20I I; 56:2 I 66-78. http://dx.doi.org/I0.1007/s I0620-0 I I-I585-2

5 lanora AAS, Memeo M, Sabba $C$ et al. Hereditary hemorrhagic telangiectasia: multi-detector row helical CT assessment of hepatic involvement. Radiology 2004; 230:250-9.

6 Faughnan M, Palda V, Garcia-Tsao G et al. International guidelines for the diagnosis and management of hereditary haemorrhagic telangiectasia. J Med Genet 20II; 48:73-87. http://dx.doi. org/10.1136/jmg.2009.069013

7 Garcia-Tsao G, Korzenik JR, Young $L$ et al. Liver disease in patients with hereditary hemorrhagic telangiectasia. N Engl Med J 2000; 343:931-6.

8 Fagel WJ, Perlberger R, Kauffmann RH. Portosystemic encephalopathy in hereditary hemorrhagic telangiectasia. Am J Med 1988; 85:858-60.

9 Chavan A, Caselitz M, Gratz K-F et al. Hepatic artery embolization for treatment of patients with hereditary hemorrhagic telangiectasia and symptomatic hepatic vascular malformations. Eur Radiol 2004; 14:2079-85.

10 Dupuis-Girod S, Ginon I, Saurin J-C et al. Bevacizumab in patients with hereditary hemorrhagic telangiectasia and severe hepatic vascular malformations and high cardiac output. JAMA 2012; 307:948-55. http://dx.doi.org//0.1001/jama.2012.250
I I Bose P, Holter JL, Selby GB. Bevacizumab in hereditary hemorrhagic telangiectasia. N Engl Med J 2009; 360:2/43-4. http://dx.doi. org/I0.1056/NEJMc090I42I

12 Young LH, Henderson KJ, White RI et al. Bevacizumab: finding its niche in the treatment of heart failure secondary to liver vascular malformations in hereditary hemorrhagic telangiectasia. Hepatology 20I3; 58:442-5. http://dx.doi.org/I0.I002/hep.26472

13 Sabbà C, Pompili M. The hepatic manifestations of hereditary haemorrhagic telangiectasia. Aliment Pharmacol Ther 2008; 28:52333. http://dx.doi.org/I0.III I/j. I365-2036.2008.03775.x

14 Lacout A, Marcy PY, El Hajjam M et al. Tranexamic acidbevacizumab synergy in HHT patients presenting pulmonary arteriovenous malformations. Med Hypotheses 2012; 79:605-6. http://dx.doi.org/I0.1016/j.mehy.2012.07.031

15 Sopeña B, Gimena B, Pérez-Rodríguez MT et al. An unusual cause of pulmonary hypertension and refractory edema. Int J Cardiol 20I I; I48:el-2. http://dx.doi.org/I0.10I6/j.ijcard.2009.0I.035

16 Trembath RC, Thomson JR, Machado RD et al. Clinical and molecular genetic features of pulmonary hypertension in patients with hereditary hemorrhagic telangiectasia. N Engl Med J 200I; 345:325-34.

17 Simonneau G, Gatzoulis MA, Adatia I et al. Updated clinical classification of pulmonary hypertension. J Am Coll Cardiol 2013; 62:D34-4I. http://dx.doi.org//0.1016/j.jacc.2013.10.029

I8 Atallah E, Durand J, Kantarjian $\mathrm{H}$ et al. Congestive heart failure is a rare event in patients receiving imatinib therapy. Blood 2007; I 10:1233-7.

19 Barst RJ, Gibbs JS, Ghofrani HA et al. Updated evidence-based treatment algorithm in pulmonary arterial hypertension. J Am Coll Cardiol 2009;54:S78-84.http://dx.doi.org/I0.1016/j.jacc.2009.04.0I7 